\title{
Dam Management With Imperfect Models: Bayesian Model Averaging and Neural Network Control
}

\author{
Paul J. Darwen \\ School of Business, James Cook University, Brisbane Campus \\ 349 Queen Street, Brisbane, Queensland, Australia \\ paul. darwen@jcub.edu.au
}

\begin{abstract}
Dam management is a controversial control problem for two reasons. Firstly, models are (by definition) crudely simplified versions of reality. Secondly, historical rainfall data is limited and noisy. As a result, there is no agreement on the "best" control policy for running a dam. Bayesian model averaging is theoretically a good way to cope with these difficulties, but in practice it degrades under two approximations: discretizing the parameter space, and excluding models with a low probability of being correct. This paper explores the practical aspects of how Bayesian model averaging with a neural network controller can improve dam management and flood control.
\end{abstract}

\section{Motivation}

A dam on a river has two functions, to store water for dry times, and to prevent flooding. Unfortunately, these two functions are diametrically opposed:

- To prevent flooding, you should gradually let out all the water from the dam, so that it can catch a future flood.

- To store water for dry times, you should never let out any water.

The dam control problem is stark: how much water should we keep in the dam, and how much should we let out? Currently there is no consensus answer.

Section 3.1 looks at the simple control policy of always letting the water level down to some fixed percentage of the dam's capacity. Section 3.3 considers a more elaborate control policy using a neural network found by expectation maximization, either:

- By finding the single model that best fits historical data (using expectation maximization again), or;

- By finding a whole distribution of plausible models with Bayesian model averaging [1], an approach which is theoretically better [4, page 175].

This paper explores the practicality of the Bayesian approach. It consumes vastly more computer time, even with two approximations: discretizing the space of models, and deleting models with sufficiently low probability of being correct. 


\section{A Dam Control Problem}

Imagine a river with a dam that has controllable release gates, so the dam's water level can be reduced to any desired level. To calibrate a rainfall model, only 100 years of historical rainfall data exist. For a future 50-year period, the two conflicting aims are to avoid either of these disasters:

- Avoid flooding, when the dam level reaches rises above $100 \%$.

- Avoid running empty, when the dam level reaches $0 \%$.

Evidence suggests that the weather in eastern Australia follows a 5-year cycle of wet and dry, with both shorter- and longer-term cycles to complicate matters [3]. To capture that, this paper makes up a stochastic rainfall function that gives river flow at time $t$ (in months) by taking random samples from a lognormal function with standard deviation $\sigma=0.1$ and mean $\mu$ given by:

$$
\begin{aligned}
\mu=\ln (2+ & (0.3299928 \times \tanh (3.3 \times \sin (t / 9.55)) \\
+ & 0.3345885 \times \tanh (3.4 \times \sin (t / 7.00)) \\
+ & 0.3354186 \times \tanh (3.5 \times \sin (t / 4.15))))
\end{aligned}
$$

Equation 1 has three flood/drought cycles with roughly equal importance, with periods of $4.15,7$, and 9.55 months. The rest is merely to make it more complicated than the simple model described next in Section 2.1.

To generate 100 years of historical data, running this rainfall function with a particular random number seed gives the historical data shown in Figure 1.

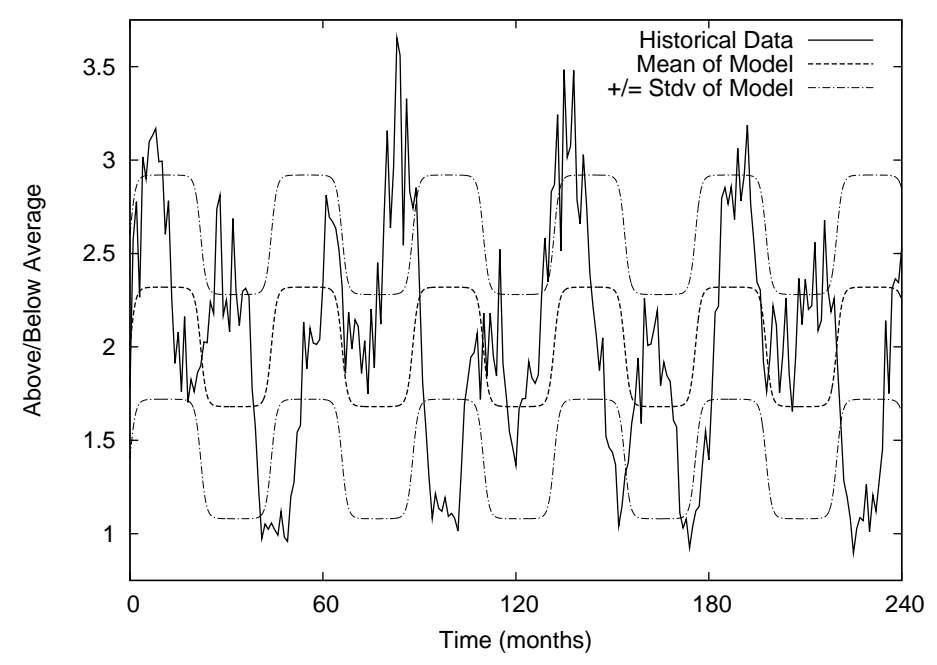

Fig. 1. The first 20 years of the historical data generated by Equation 1, overlaid with the single best-fit model with parameters $w 0=0.32$ and $w 1=6.9964$ in Equation 2 . 


\subsection{A Crude Model}

All rainfall models are simpler than the real world, and here the model in Equation 2 is too simple to capture the actual rainfall function in Equation 1. Here, a simple stochastic model has the following form, with mean river flow $\mu$ set to:

$$
\mu=2.0+w 0 * \tanh (4.0 * \sin (t / w 1))
$$

That is, the model assumes a cycle from flood to drought, where the two model parameters are $w 0$ the amplitude and $w 1$ the duration of that cycle, and it predicts the river's mean flow at time $t$ in months. An example of this model for a particular choice of parameters $w 0$ and $w 1$ is in Figure 1.

To give the model a stochastic flavour, the prediction is a random sampling from a normal distribution with mean given by Equation 2 and standard deviation $\sigma=0.6$. Any negative predictions are set to zero.

\subsection{A Control Function}

A popular control policy is to set a single parameter, namely how much of the dam's capacity to fill with water, with the unused capacity being a "flood compartment". This policy will be evaluated later in Section 3.1.

A more elaborate controller could be a neural network, and this paper uses the simplest kind: a sigmoid function equivalent to a one-node neural network.

$$
\theta\left(u_{0}, u_{1}, x, t\right)=\frac{1}{1+e^{u_{0}+u_{1} x}}
$$

The sigmoid function $\theta$ in Equation 3 has bias $u_{0}$ and weight $u_{1}$, and takes input $x\left(w_{0}, w_{1}, t\right)$ the water level of the dam at time $t$ as predicted by a model with parameters $w_{0}$ and $w_{1}$ from Equation 2. The sigmoid function $\theta$ is the desired water level that the dam should be lowered to. Of course, if the dam's water level is already less than that, then no water need be released.

As a sigmoid function can take an S-shape, the general aim of this controller function is to suggest a lower dam level if wet weather is predicted, and to suggest a higher dam level if dry weather is predicted.

\subsection{The Single Best-Fit Model versus Bayesian Model Averaging}

A popular approach for finding a controller is to calibrate the model's parameters to the historical data, and then calibrate a controller to that single best-fit model.

To find that single best-fit model, expectation maximization is a popular approach: it finds the model that has the highest probability of being correct, given the data [2]. For the historical data in Figure 1, the most probable model has $w 0=0.32$ and $w 1=6.9964$ in Equation 2. Figure 1 shows this model.

The problem with the best-fit model is that the "highest probability of being correct" often turns out to be disappointingly improbable. With so little historical data, many other models are less probable, but still plausible. 


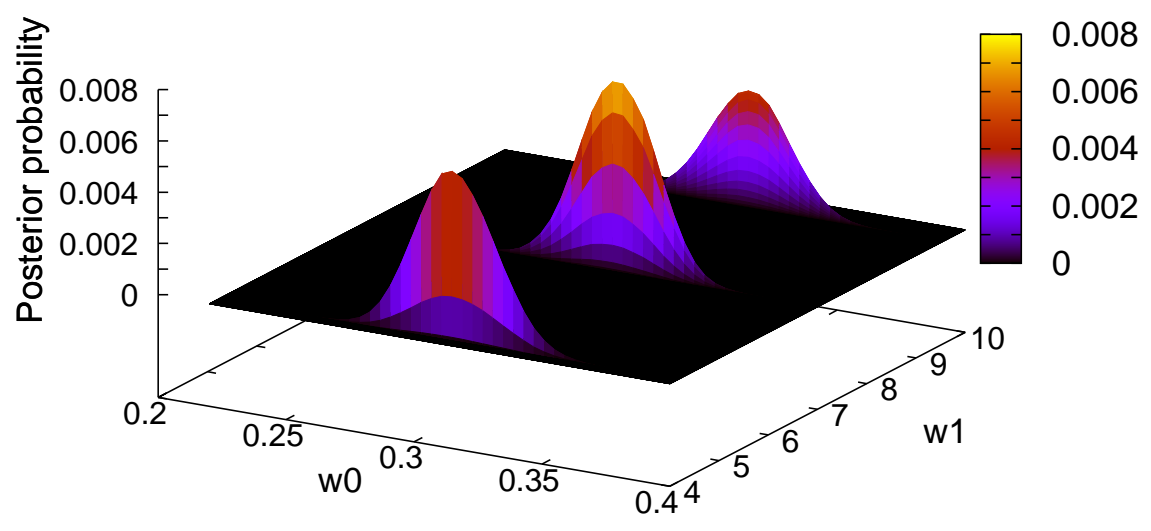

Fig. 2. The probability distribution of plausible models. The single most-probable model is the one at the peak of the highest hill.

In the Bayesian model averaging approach, the aim is to iterate over all those plausible models to find the probability that a model is correct, given the historical data. Figure 2 shows this probability distribution for our test problem. The highest point in that distribution represents the most probable model, at parameters $w 0=0.32$ and $w 1=6.9964$. But picking the highest point in the probability distribution (i.e., the best-fit model) ignores all those other lessprobable models that are still plausible, throwing away much of the information in the historical data.

In this paper, the problem is not to find the single "best" model, but instead to come up with an adequate controller despite having a too-simple model.

\subsection{Two Approximations to Bayesian Model Averaging}

Bayesian model averaging is theoretically the better method [4, page 175], but that proof assumes a continuous world. In the messy world of numerical approximations, there are trade-offs. This section describes two approximations.

Firstly, the distribution of models in Figure 2 may look smooth, but it uses a discretized grid to step through the model parameters. The $w_{0}$ axis has step size 0.004 and $w_{1}$ has a smaller step size of 0.0012 to capture those narrow sails. These step sizes were tuned by hand, to be small enough make Figure 2 smooth.

Secondly, even with discretized model parameters, there are still a great many models with non-zero probability of being correct. Figure 3 counts how many models there are, as we descend from the peaks of Figure 2. Figure 3 can be extended to the left side without limit, so if you really want every single model with non-zero probability of being correct, there is essentially no limit. Rather than enumerate every single model, a reasonable approximation is to use some minimum cutoff, and ignore the low-probability models. From Figure 3, a cutoff of $10^{-6}$ will take the 1,489 most probable models. 


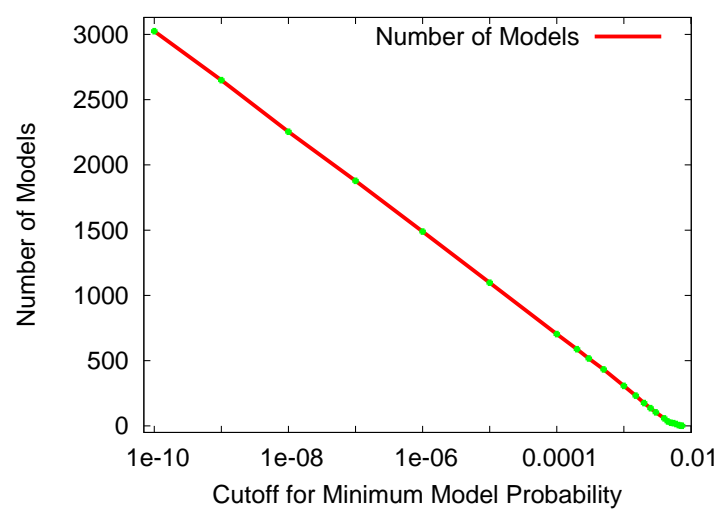

Fig. 3. There are an unlimited number of low-probability models, so this paper uses a cutoff of $10^{-6}$, which makes for 1,489 models.

\section{Results}

\subsection{A Fixed Level Is Not A Good Policy}

A simple control policy is to gradually release water from the dam until the level is down to some fixed percentage of capacity, so the unused dam space can be a "flood compartment". This section evaluates that kind of policy.

Take the single model that best fits the historical data, and use it to evaluate various fixed levels by doing Monte Carlo simulation of many possible 50-year futures. The peak of the solid line in Figure 4 shows that the single best-fit model predicts a fixed level of $71.65 \%$ would be the best water level to keep the dam at, and doing so should prevent disaster (either the dam over-filling, or running empty) with a probability of $99.35 \%$ over the next 50 years. Sounds pretty good, if you trust that single best-fit model!

Unfortunately, the dashed line in Figure 4 uses the true, unseen function that generates rainfall (from Equation 1) to evaluate the true probability of success. If you run the dam at a constant water level of $71.65 \%$ as suggested by the single best-fit model, then your true probability of success is a lousy $9.4 \%$, much less than the $99.35 \%$ that the single best-fit model has led you to believe.

\subsection{A Neural Network Controller from the Single Best-Fit Model}

Take the single best model, which is the peak of Figure 2), and use it to find the best controller function. That single best model says its best controller should have a success rate of about $98 \%$, which sounds pretty safe.

Unfortunately, running that controller through 400,000 simulated futures using the true rainfall function gives a true success rate of only $32.3 \%$. The actual success rate is worse because of the simplicity of the model's functional form, and the limited historical data. So for this approach, you have unwittingly doomed your city with a $67.7 \%$ chance of disaster some time in the next 50 years, even though your best-fit model predicts only a $2 \%$ chance of disaster. 


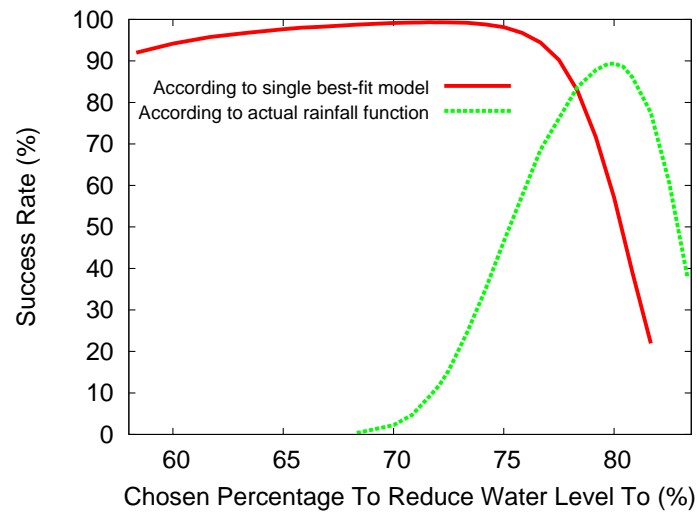

Fig. 4. A simple control policy is to gradually release water to bring the level down to some fixed percentage. Using the single model that best fits the historical data, this shows how well the fixed-level approach works according to that single best model, and according to the actual, unseen rainfall function. Keeping the water level at $71.65 \%$ (as the best-fit model suggests) would give a real success rate of only $9.4 \%$.

\subsection{A Neural Network Controller from Bayesian Model Averaging}

Bayesian model averaging generates the whole distribution of models shown in Figure 2. This paper discretizes that distribution and then ignores the lowprobability models, as described in Section 2.4.

So here, the best controller is the one that performs best, according to the weighted vote of all 1,489 models. The weighting is according to each model's probability of being correct. This approach takes about 1,498 times as much computer time as using the single best-fit model. The best controller should get a success rate of $98 \%$, according to those models, showing that the controller function is not the bottleneck - whatever the model(s), there is a controller which supposedly will have a $98 \%$ success rate, according to those models.

Taking that winning controller from Bayesian model averaging, and running it through 400,000 simulated 50-year futures using the true rainfall function gives a true success rate of $45.796 \%$. That's better than the $32.319 \%$ from the single best-fit model in Section 3.2, and much better than the lousy $9.4 \%$ from using a fixed level back in Section 3.1. These differences are statistically significant.

The single best-fit model gives a controller at the parameters $u_{0}=-2.4375$ and $u_{1}=1.546875$. In contrast, the controller from Bayesian model averaging is at $u_{0}=-2.83984375$ and $u_{1}=+1.9140625$, a substantial difference.

Equation 1 describes the (usually unseen) rainfall function. So this section cheats and uses that true function to find the ideal controller: it's at $u_{0}=$ -5.5 and $u_{1}=+3.9$ and gives a success rate of $98.8 \%$ on the true rainfall function. Again, all controllers get about $98 \%$ on their choice of models, so it's not controller optimization that matters here. Figure 5 shows all three controllers in parameter space - the Bayesian approach's controller is closer to the ideal. 


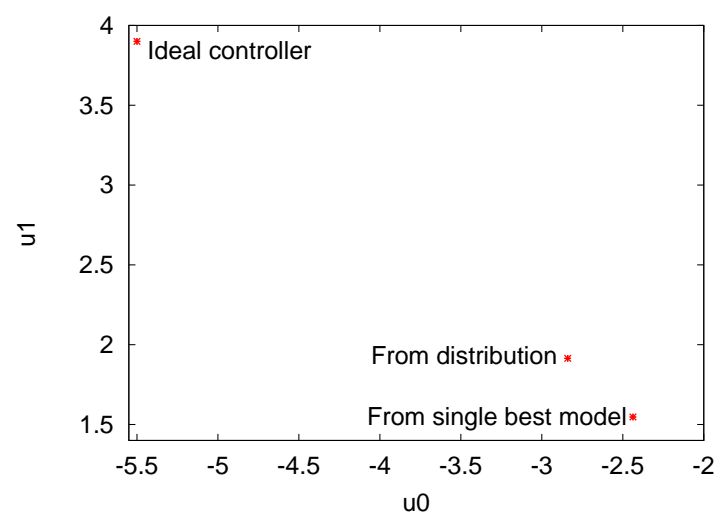

Fig. 5. The two controller functions, one found from the single best model, and the other from a Bayesian distribution of all plausible models, are shown here with the ideal controller, found by using the (usually unseen) function that generates rainfall. The Bayesian approach is closer to the ideal.

\section{Discussion and Conclusion}

Models are merely simplified, abstracted version of the real thing. A more realistic model would have more than the 2 parameters in Equation 2. However, models with a many free parameters require more data. Any practical rainfall model cannot have a large number of free parameters, due to the shortage of historical data. This avoids a combinatorial explosion from a high-dimensional space of model parameters. So long as the space of plausible models has reasonably low dimensions, then iterating through that space of models should be feasible for the Bayesian model averaging approach.

In this problem, the complexity of the neural network controller and the algorithm for optimizing that controller were not the bottlenecks that prevent success - in fact, even the simple controller used here was good enough for a $98 \%$ success rate. The bottleneck is that the model is too simple. For such models, Bayesian model averaging is a practical way do dam management and similar control problems.

\section{References}

1. Hoeting, J.A., Madigan, D., Raftery, A.E., Volinsky, C.T.: Bayesian model averaging: A tutorial. Statistical Science 14(4), 382-417 (1999)

2. Jeffreys, H.: Theory of Probability. The International Series of Monographs on Physics, Oxford University Press, 1 edn. (1939)

3. Mazzarella, A., Giuliacci, A., Liritzis, I.: On the 60 -month cycle of multivariate ENSO index. Theoretical and Applied Climatology 100, 2327 (2010)

4. Mitchell, T.M.: Machine Learning. McGraw-Hill, New York (Mar 1997) 